\title{
Background independent quantum mechanics and gravity
}

\author{
Djordje Minic* and Chia-Hsiung Tze ${ }^{\dagger}$ \\ Institute for Particle Physics and Astrophysics, Department of Physics, Virginia Tech, Blacksburg, Virginia 24061, USA
}

(Received 30 May 2003; published 29 September 2003)

\begin{abstract}
We argue that the demand of background independence in a quantum theory of gravity calls for an extension of standard geometric quantum mechanics. We discuss a possible kinematical and dynamical generalization of the latter by way of a quantum covariance of the state space. Specifically, we apply our scheme to the problem of a background independent formulation of matrix theory.
\end{abstract}

DOI: 10.1103/PhysRevD.68.061501

PACS number(s): 04.60.- m, 11.25.Yb

The quest for a consistent, unified quantum theory of matter and gravity remains very much an open issue, despite great progress in string theory [1] and other approaches to quantum gravity [2]. At the outset either quantum mechanics (QM) or general relativity (GR) or both should give way to a new substructure. From the predominant viewpoint in string theory it is GR that needs replacing, while QM is complete by itself. This stand is well motivated when GR is taken as valid only at low energy scales [1]. In fact, the most general diffeomorphism invariant effective action is derivable from the consistency requirements (i.e., conformal invariance) of a perturbative string theory. Alas, a nonperturbative, background independent understanding of this remarkable fact is still lacking. Most of the other attempts at quantizing gravity [2], while stressing the relational, background independent nature of GR (and thus arguing against the effective field theoretic point of view and for a nonstandard approach to quantization of gravity) still keep to the canonical structure of QM. ${ }^{1}$

What is the physical rationale for changing the canonical quantum mechanical structure? And if such a rationale is found, is it theoretically or empirically compelling? In the negative, is not the canonical structure somehow unique, and if so, what does this imply for the foundational issues of quantum theory, of a theory of quantum gravity, in particular?

This paper puts forth a radical but, in our view, a justified approach to extending QM. Motivated by the physical requirement of background independence and the need to make room for gravity at the quantum level, we are led to a rather drastic extension of standard QM: to wit, we modify both its dynamics and kinematics, and thereby the very symplectic and Riemannian structures that underlie its geometric foundations. ${ }^{2}$ The upshot of our proposal is that the space of quantum states (events) becomes dynamical and that the dynamical geometric information is described in terms of a nonlinear diffeomorphism invariant theory, in such a way

\footnotetext{
*Email address: dminic@vt.edu

†Email address: kahong@vt.edu

${ }^{1}$ For a recent critical discussion on the status of canonical QM in quantum gravity, see, for example [3].

${ }^{2}$ Our present discussion is far more general than our recent geometric formulation of Nambu quantum mechanics [4], motivated by the covariance problem in matrix theory.
}

that the space of quantum events is nonlinearly inter-related with the generator of quantum dynamics - the Hamiltonian.

We briefly review the key features of geometric standard QM [5] (for reviews of this approach consult [6-10]). Pure states are points of an infinite dimensional Kahler manifold $\mathcal{P}(\mathcal{H})$, the complex projective space of the Hilbert space $\mathcal{H}$. Equivalently, $\mathcal{P}(\mathcal{H})$ is a real manifold with an integrable almost complex structure $J$. As such it has a Kahler metric given by $\langle\psi \mid \phi\rangle$, the Hermitian inner product of two states $\langle\psi|$ and $|\phi\rangle$ in $\mathcal{H}$, the Riemannian metric $g(\psi, \phi)$ $=g(J \psi, J \phi)=2 k \operatorname{Re}(\langle\psi \mid \phi\rangle)$, which is uniquely the CayleyFubini-Study metric [7,9]. The associated symplectic 2-form $\omega(\psi, \phi)=2 k \operatorname{Im}(\langle\psi \mid \phi\rangle)$ with $k=\hbar=2 / c$ with $h$ being Planck's constant and $c$ the constant holomorphic sectional curvature (CHSC) of $\mathcal{P}(\mathcal{H})$. While the role of the symplectic structure is well known, the Riemannian structure, notably absent in classical phase space, is the key player and as the metric structure on $\mathcal{P}$ embodies the information about purely quantum properties, such as time evolution, uncertainty relations, entanglement, and the measurement process. The correspondence with the operatorial formalism is as follows: An observable $A=\langle\hat{A}\rangle$, defined as the expectation value of a Hermitian linear operator $\hat{A}$, is a real valued differentiable function on $\mathcal{P}$, belonging to a special class of Kahlerian functions. This contrasts sharply with classical Hamiltonian dynamics where any function of the canonical variables is an observable. ${ }^{3}$ The derivative of such an $A$ vanishes at an "eigenstate" with the value of $A$ at such a point giving the "eigenvalue."

The evolution of states, i.e., the Schrödinger equation is given by the symplectic flow generated by a Hamiltonian $H$ of any given system. Let a pure state be $\psi=\Sigma_{a} e_{a} \psi_{a}$, where the $\psi_{a}$ are the coefficients of $\psi$ in an orthonormal eigenbasis $\left\{e_{a}\right\}$ of $H$. Let $q^{a}=\sqrt{2 \hbar} \operatorname{Re} \psi_{a}$ and $p_{a}=\sqrt{2 \hbar} \operatorname{Im} \psi_{a}$ with the $\left(q^{a}+i p_{a}\right)$ being the homogeneous coordinates for $\mathcal{P}$. The symplectic structure on $\mathcal{P}$ is given by the closed, nondegenerate 2-form $\omega^{(2)}=d p_{a} \wedge d q^{a}, d \omega^{(2)}=0$. The Poisson bracket is defined as usual: $\{f, g\}=\left(\partial f / \partial p_{a}\right)\left(\partial g / \partial q^{a}\right)$ $-\left(\partial f / \partial q^{a}\right)\left(\partial g / \partial p_{a}\right) \equiv \omega^{a b}\left(\partial f / \partial X^{a}\right)\left(\partial g / \partial X^{b}\right)$, where $\omega^{a b}$ is the inverse of $\omega^{(2)}$ and the $X^{a}=\left(p_{a}, q^{a}\right)$ form a set of canonical coordinates. The Schrödinger equation, with $h$

\footnotetext{
${ }^{3}$ Such a possibility in the context of a generalized QM was analyzed by Weinberg [6].
} 
$=\langle\hat{H}\rangle$, is then simply Hamilton's equations $d p_{a} / d t$ $=\left\{h, p_{a}\right\}, \quad d q^{a} / d t=\left\{h, q^{a}\right\} . \quad$ Here $\quad h=\frac{1}{2} \Sigma_{a}\left[\left(p^{a}\right)^{2}\right.$ $\left.+\left(q_{a}\right)^{2}\right] \omega_{a}, \omega_{a}$ being the energy eigenvalues. An observable $O$ will then evolve as $d O / d t=\{h, O\}$. The expectation values of commutators of operators acting on $\mathcal{H}$ are precisely the Poisson brackets of the corresponding Kahlerian functions The inner product determines the flat metric on the Hilbert space $d S^{2}=\Sigma\left[\left(d q_{a}\right)^{2}+\left(d p_{a}\right)^{2}\right]=\delta_{a b} d X^{a} d X^{b}$, where $X^{a}=\left(q^{a}, p^{a}\right)$.

The Born rule $\psi^{*} \psi=1=(1 / 2 \hbar) \Sigma_{a}\left[\left(p^{a}\right)^{2}+\left(q_{a}\right)^{2}\right]=1$ implies that $\psi$ and $e^{i \alpha} \psi$ are to be identified. For finite $n$, we then have as the space of rays in $\mathcal{H}=C^{n+1}$, the complex projective space $C P(n)$, the base space of the complex Hopf line bundle of the sphere $S^{2 n+1}$ over $C P(n)=U(n$ $+1) / U(n) \times U(1)$ with its $U(1)$ fiber, the group of complex phases in QM. Thus QM can be viewed as a classical Hamiltonian system, albeit a very special one with, as its phase space, the nonlinear, rich and "huge" projective Hilbert space $C P(n)$ with $n=\infty$ generically [8] and $U(n)$ as the unitary group of quantum canonical transformations. The unique Riemannian metric on $C P(n)$, induced from the inner product of $\mathcal{H}$, is the Fubini-study (FS) metric, $d s_{12}^{2}$ $=4\left(1-\left|\left\langle\psi_{1} \mid \psi_{2}\right\rangle\right|^{2}\right) \equiv 4(\langle d \psi \mid d \psi\rangle-\langle d \psi \mid \psi\rangle\langle\psi \mid d \psi\rangle)$ as explicitly discussed in $[7,9,11]$. The Heisenberg uncertainty relations arise from such a metric of $C P(n)$ whose local properties also yield a generalized energy-time uncertainty relation [9]. The probabilistic (statistical) interpretation of QM is thus hidden in the metric properties of $\mathcal{P}(\mathcal{H})$. The unitary time evolution is related to the metrical structure [9] with Schrödinger's equation in the guise of a geodesic equation on $C P(n)=U(n+1) / U(n) \times U(1): d u^{a} / d s+\Gamma_{b c}^{a} u^{b} u^{c}$ $=(1 / 2 \Delta E) \operatorname{Tr}\left(H F_{b}^{a}\right) u^{b}$ for the FS metric $g_{a b}^{F S}$ with the canonical curvature 2-form $F_{a b}$ valued in the holonomy gauge group $^{4} U(n) \times U(1)$. Here $\Delta E^{2}=\left\langle H^{2}\right\rangle-\langle H\rangle^{2}$ as in [9]. Also, $u^{a}=d z^{a} / d s$, where $z^{a}$ denote the complex coordinates on $C P(n)$ and $\Gamma_{b c}^{a}$ is the connection obtained from the FS metric. The affine parameter $s$ is determined by the $C P(n)$ metric. As underscored by Aharonov and Anandan [9], time measurement in the evolution of a given system reduces to that of distance on $C P(n)$. In particular, $\hbar d s=2 \Delta E d t$. Such an expression naturally invokes a relational interpretation of time in QM. Even more striking is the fact that the geometric interpretation of probability as the geodesic distance on $C P(n)$ is directly related to the definition of the evolution parameter $t$ ! In the above geodesic Schrödinger equation, $H$ appears as the "charge" of an effective particle moving with a "velocity" $u$ in the background of the "Yang-Mills" field $F_{a b}$. Finally, given a curve $\Gamma$ in the projective Hilbert space $\mathcal{P}$, the geometric (Berry) phase [13] is given by [9] $\int_{\Sigma} d p_{a}$

\footnotetext{
${ }^{4}$ The best way to understand this geodesic equation is to realize that the Hamiltonian flow on the Hilbert space is a Killing flow [7]. So the Schrödinger equation on $C P(n)$ is the geodesic equation projected from the $U(n+1)$ bundle down to the base space $C P(n)$ with the fibre $U(n) \times U(1)$. This is just the Kaluza-Klein reduction that leads to the geodesic equation of this type, better known as the Wong equation $[9,12]$.
}

$\wedge d q^{a}$, where $\Sigma$ has as its boundary $\Gamma$. As a symplectic area enclosed by $\Sigma$, this phase depends solely on the geometry of the inner product and is both independent of the Hamiltonian and the equation of motion if and only if the latter is first order in time.

Next we draw attention to a simple calculation of $d s_{12}^{2}$ for the Gaussian coherent state $\psi_{l}(x) \sim \exp \left[-(\vec{x}-\vec{l})^{2} / \delta l^{2}\right]$, which, using the convolution property of Gaussian integrals, yields the natural metric in the configuration space, namely, $d s^{2}$ $=d \vec{l}^{2} / \delta l^{2}$. So, wherever the configuration space coincides with space, the natural metric on $C P(n)$ in the $\hbar \rightarrow 0$ limit gives a spatial metric [9]. It is this important insight which is the springboard for our proposed background independent generalization of standard $Q M$. For a generalized coherent state, the FS metric reduces to the metric on the corresponding group manifold [10].

Given the Riemannian structure of QM and the observed connection between the FS and the spatial metrics, it behooves us to inquire if a more general Riemannian structure of space can be induced from a more flexible state space than $C P(n)$. Specifically, let $l$ in the above metric computation be mapped to $t \rightarrow k(l)$. The corresponding expression for the spatial metric results from the overlap of two Gaussians $\psi_{k(l)}(x) \sim \exp \left(-[x-k(l)]^{2} / \delta k(l)^{2}\right)$, which in turn follows from $\int d x g_{\psi_{l}, \psi_{l+d l}} \psi_{l}^{*} \psi_{l+d l}$, where the "quantum metric" reads $g_{\psi_{l}, \psi_{l+d l}} \equiv \psi_{k}^{*} \psi_{k+d k} / \psi_{l}^{*} \psi_{l+d l}$. Clearly the transformation that takes $\psi_{l} \rightarrow \psi_{k(l)}$ is not in general unitary. If we insist on the desired relation between the quantum metric and an arbitrary metric on the classical configuration space, then the kinematics of QM must be altered. Moreover if the induced classical configuration space is to be the actual space of spacetime, only a special quantum system will do. We are thus induced to make the state manifold suitably flexible by doing general relativity on it. The resultant metric on the Hilbert space is generally curved with its distance function modified, an extended Born rule and hence a new meaning to probability. By insisting on diffeomorphism invariance in the state space and on preserving the desirable complex projective properties of Cartan's rank 1 symmetric spaces such as $C P(n)$, we arrive at the ensuing coset state space $\operatorname{Diff}(\infty, C) / \operatorname{Diff}(\infty-1, C) \times \operatorname{Diff}(1, C)$ as the minimal phase space candidate for a background independent QM. In summary, the axioms of standard geometric QM are enlarged as follows.

(1) The state space $C P(\infty)$ is extended to $\operatorname{Diff}(\infty, C) / \operatorname{Diff}(\infty-1) \times \operatorname{Diff}(1, C)$ deriving from the generalized inner product

$$
d S^{2}=\sum h_{a b}\left[\left(d q_{a}\right)^{2}+\left(d p_{a}\right)^{2}\right] \equiv h_{a b} d X^{a} d X^{b},
$$

where $h_{a b}$ is Hermitian. The "Born rule" now reads

$$
\frac{1}{2} \sum_{a, b} h_{a b}\left[\left(p^{a} p^{b}\right)+\left(q^{a} q^{b}\right)\right]=1 .
$$

These equations provide the metric relation on and the geometrical shape(s) of the new state space, and implicitly de- 
fines $\hbar$. The probabilistic interpretation lies in the definition of geodesic length on the new space of quantum states (events). The relation $\hbar d s=2 \Delta E d t$ gives meaning to the "evolution parameter" $t$ ! Notably different metrics imply different evolution parameters with $t$ relational and akin to the "multifinger time" of GR [14]. Given the $X$ space, we can introduce a natural $\operatorname{Dif} f(1, C)$ map, $X \rightarrow f(X)$. The $\operatorname{Diff}(1, C)$ identification of the points on the submanifold determined by the Born rule defines the generalized projective Hilbert manifold.

(2) The observables are functions of the natural distance on the quantum phase space $h_{a b} X^{a} X^{b}, O=O\left(h_{a b} X^{a} X^{b}\right)$. They reduce to the usual ones when the Riemannian structure is canonical. More explicitly

$$
O=\sum_{a, b} o_{a} h_{a b} X^{a} X^{b},
$$

where the eigenvalue $o_{a}$ is given as (see [6])

$$
\frac{d O}{d X^{a}}=o_{a} \omega_{a b} X^{b} .
$$

Here the symplectic form $\omega^{a b}$ as well as $O$ depend on the invariant combination $h_{a b} X^{a} X^{b}$.

(3) The temporal evolution equation reads (see footnote 6)

$$
\frac{d u^{a}}{d \tau}+\Gamma_{b c}^{a} u^{b} u^{c}=\frac{1}{2 \Delta E} \operatorname{Tr}\left(H F_{b}^{a}\right) u^{b},
$$

where now $\tau$ is given through the metric $\hbar d \tau=2 \Delta E d t$, as in the original work of Aharonov and Anandan [9]. $\Gamma_{b c}^{a}$ is the affine connection associated with this general metric $g_{a b}$ and $F_{a b}$ is a general curvature 2-form in $\operatorname{Diff}(\infty-1, C)$ $\times \operatorname{Diff}(1, C)$.

Next we reformulate geometric QM in the above background independent (BI) setting. Due to the $\operatorname{Diff}(\infty, C)$ symmetry, "coordinates" $z^{a}$ (i.e., quantum states) make no sense physically, only quantum events do, which is the quantum counterpart of the corresponding statement on the meaning of spacetime events in GR. Probability is generalized and given by the notion of diffeomorphism invariant distance in the space of quantum configurations. The dynamical equation is a geodesic equation on this space. Time, the evolution parameter in the generalized Schrödinger equation, is not global and is given in terms of the invariant distance. Our basic starting point of a background independent QM (BIQM) is to notice that the evolution equation (the generalized Schrödinger equation) as a geodesic equation, can be derived from an Einstein-like equation with the energymomentum tensor determined by the holonomic non-Abelian field strength $F_{a b}$ of the $\operatorname{Diff}(\infty-1, C) \times \operatorname{Diff}(1, C)$ type and the interpretation of the Hamiltonian as a charge. Such an extrapolation is logical since $C P(n)$ is an Einstein space; its metric obeying Einstein's equation with a positive cosmological constant given by $\hbar: R_{a b}-\frac{1}{2} g_{a b} R-\lambda g_{a b}=0$. The Ricci curvature of $C P(n)$ is $R_{a b} \equiv(n+1) / \hbar g_{a b}=\frac{1}{2} c(n$ $+1) g_{a b}$, where $c$ is the CHSC of $C P(n)$ given by $c=2 / \hbar$.
The geodesic equation (5) follows from the conservation of the energy-momentum tensor $\nabla_{a} T^{a b}=0$ with $T_{a b}$ $=\operatorname{Tr}\left[F^{a c} g_{c d} F^{c b}-\frac{1}{4} g_{a b} F_{c d} F^{c d}+(1 / 2 \Delta E) H u_{a} u_{b}\right]$ by way of the usual GR argument (e.g. [14], Chap. 20). With quantum gravity in mind, we set $\Delta E$ to the Planck energy $E_{p}$, the proper deformation parameter. When $E_{p} \rightarrow \infty$ we recover the usual flat metric on the Hilbert space or the FS metric on the projective Hilbert space. Since both the metrical and symplectic data are also contained in $H$, we have here the advertized nonlinear "bootstrap" between the space of quantum events and the dynamics. The diffeomorphism invariance of the new phase space suggests the following dynamical scheme for the BIQM:

$$
R_{a b}-\frac{1}{2} g_{a b} R-\lambda g_{a b}=T_{a b},
$$

with $T_{a b}$ given as above [as determined by $F_{a b}$ and the Hamiltonian (charge) $H$ ]. Furthermore

$$
\nabla_{a} F^{a b}=\frac{1}{2 \Delta E} H u^{b} .
$$

The last two equations imply via the Bianchi identity a conserved energy-momentum tensor, $\nabla_{a} T^{a b}=0$. The latter, taken together with the conserved "current" $j^{b} \equiv(1 / 2 \Delta E) H u^{b}$, i.e., $\nabla_{a} j^{a}=0$, implies the generalized geodesic Schrödinger equation. So Eqs. (6) and (7), being a closed system of equations for the metric and symplectic form on the space of events, define our BIQM. We emphasize once again that in the limit $E_{p} \rightarrow \infty$ we recover the usual structure of linear QM. Notice that in this limit the metric and the symplectic structure do not depend on the Hamiltonian, which is the case in ordinary quantum mechanics. By imposing the conditions of homogeneity and isotropy on the metric (that is the requirement of the maximum number of Killing vectors) the usual rigid structure of quantum mechanics is recovered [7]. Moreover this limit does not affect the geodesic equation $d u^{a} / d \tau+\Gamma_{b c}^{a} u^{b} u^{c}=(1 / 2 \Delta E) \operatorname{Tr}\left(H F_{b}^{a}\right) u^{b}$ due to the relation $\hbar d \tau=2 \Delta E d t$. As such our formulation offers a tantalizing nonlinear linkage between the metric and symplectic data embodied in $H$ and the quantum metric and symplectic data. The space of quantum events is dynamical paralleling the dynamical role of spacetime in GR, as opposed to the rigid, absolute state space of standard QM. This is then, in our view, the price of quantum background independence. To draw more concrete consequences of this kinematics made dynamical, we next specify a quantum system with its $H$. The configuration space of the quantum metric defines a "superspace" (as in canonical GR [15]) and the dynamics on it presumably select a particular background.

We now demand that the configuration space metric be the actual physical spatial metric. The suitable quantum system must then have a very special configuration space and should describe a quantum theory of gravity. Specifically, we seek a canonical QM of a nonperturbative form of quantum gravity in a fixed background, with a well defined perturbative limit and a configuration space being the actual space. The only example we know of fulfilling these criteria is matrix theory [16]. (The latter is also "holographic" [17], in the 
sense of mean-field theory. ${ }^{5}$ ) As with other roads to quantum gravity, matrix theory which leaves QM intact, suffers from the problem of background dependence [19-21]. ${ }^{6}$

In implementing our scheme, we assume that the metric on the transverse space is encoded in the metric on the quantum state space. Then we take the matrix theory Hamiltonian in an arbitrary background and insert it into the defining equations of the above BIQM. The evolution of our system then reads $d u^{a} / d \tau+\Gamma_{b c}^{a} u^{b} u^{c}=\left(1 / 2 E_{p}\right) H_{M} F_{b}^{a} u^{b}$, where $H_{M}$ is the matrix Hamiltonian $[i, j$ denote the transverse space indices $(i=1, \ldots, 9), R$ is the extent of the longitudinal 11th direction]

$$
\begin{aligned}
H_{M}= & R \operatorname{Tr}\left\{\frac{1}{2} P^{i} P^{j} G_{i j}(Y)+\frac{1}{4}\left[Y^{i}, Y^{l}\right]\left[Y^{k}, Y^{j}\right] G_{i j}(Y) G_{l k}(Y)\right\} \\
& + \text { fermions. }
\end{aligned}
$$

Here $P^{i}$ is the conjugate momentum to $Y^{i}(N \times N$ Hermitian matrices) given a symplectic form $\omega$. (We adopt the symmetric ordering of matrices, see [20].) Given this expression for $H_{M}$ the general equations (6) and (7) then define a background independent matrix theory (BIMT). Note that in Eqs. (6) and (7), $a, b$ denote the indices on the quantum space of states, whose span is determined by the dimension of the Hilbert space of matrix theory, given in terms of $N$.

So the time of BIMT is manifestly not global, but is defined by the invariant distance on the space of quantum events. The light-front (light-cone) $\mathrm{SO}(9)$ symmetry is only "local" (in the sense of the generalized quantum phase space). SUSY (supersymmetry) is generally broken since generically, we have no background which admits globally defined supercharges. Only "locally" (again in the sense of the generalized quantum space) we may talk about the correspondence between the moduli space of the matrix theory SUSYQM and the transverse space [16].

As to the longitudinal coordinate (and longitudinal momentum, given in terms of $N / R$ [16]), they can be made dynamical in our proposal. The rank $N$ of the matrices implicitly defines the size of the Hilbert space, which is seem-

\footnotetext{
${ }^{5}$ The relationship between holography, unitarity, and diffeomorphism invariance was explored in [18].

${ }^{6} \mathrm{We}$ should mention here that different arguments for revising quantum mechanics in the framework of quantum gravity have been advanced, for example, in [22-24].
}

ingly fixed (the dimension of the index space is fixed.) On the other hand, one of the fundamental features of matrix theory is that of being automatically second quantized; it encodes the Fock space $\left\{n_{k}\right\}$ in terms of block diagonal $n_{k}$ $\times n_{k}$ matrices [16]. Taking cue from this defining feature, we promote the points on the quantum phase space into Hermitian matrices. This is the final ingredient in our proposal. In practice, the $u^{a}$ 's appear as Hermitian matrices in the defining equations (6) and (7). So the rank of matrix-valued noncommuting transverse coordinates $Y^{i}(N)$ is made dynamical by turning the coordinates $z^{a}$ of our background independent quantum phase space into noncommutative objects. The asymptotic causal structure (and thus a covariant background independent structure) only emerges in the matrix theory limit [16], $N \rightarrow \infty, R \rightarrow \infty$ while keeping $N / R$ fixed. The above defining dynamical equations (6) and (7) can also be cast in the context of Connes' noncommutative geometry [25]. We will discuss this topic in a separate longer publication which will elaborate the contents of this paper [26].

In closing, the gist of our proposal lies in the nonlinear interconnection between the metric $\left(G_{i j}\right)$ and symplectic data $\left(\Omega_{i j}\right)$ contained in the Hamiltonian $H$ and the quantum metric $\left(g_{a b}\right)$ and symplectic data $\left(\omega_{a b}\right.$, or equivalently, $\left.F_{a b}\right)$. This nonlinear connection may well explain how (a) different degrees of freedom are associated with different backgrounds and (b) how the observed four-dimensional spacetime background dynamically emerges in matrix theory, the pregeometry being the dynamical stochastic geometry of the space of events. Furthermore we can not but ponder the fascinating possibility that the very form of the matrix theory Hamiltonian $H_{M}$ is already encoded in the nontrivial topological structure of the space of quantum events. This may be so if the latter manifold is nonsimply connected and is noncommutative. ${ }^{7}$

We are indebted to V. Balasubramanian, I. Bars, J. de Boer, L. N. Chang, L. Freidel, J. Gates, E. Gimon, M. Günaydin, V. Jejjala, D. Kabat, N. Kaloper, R. Leigh, D. Marolf, F. Markopoulou, C. Nappi, J. Polchinski, L. Smolin, A. Schwarz, T. Takeuchi, W. Taylor, and R. Zia for correspondence, comments, and discussions.

\footnotetext{
${ }^{7}$ This in complete analogy with the concept of "charge without charge" of the Einstein-Maxwell system of equations in vacuum, as discussed by Misner and Wheeler [27].
}

[1] M.B. Green, J.H. Schwarz, and E. Witten, Superstring Theory (Cambridge University Press, Cambridge, 1987), Vols. 1 and 2; J. Polchinski, String Theory (Cambridge University Press, Cambridge, 1998), Vols. 1 and 2.

[2] For recent reviews on loop quantum gravity consult, for example, T. Thiemann, gr-qc/0210094; A. Ashtekar, gr-qc/0112038; L. Smolin, hep-th/0303185; for a historical perspective and a discussion of other approaches consult, C. Rovelli, gr-qc/0006061.

[3] C.J. Isham, quant-ph/0206090.
[4] D. Minic and C.H. Tze, Phys. Lett. B 536, 305 (2002); the problem of a covariant formulation of matrix theory is explained in H. Awata, M. Li, D. Minic, and T. Yoneya, J. High Energy Phys. 02, 013 (2001); D. Minic, hep-th/9909022; hep-th/0009131.

[5] T.W. Kibble, Commun. Math. Phys. 65, 189 (1979).

[6] S. Weinberg, Ann. Phys. (N.Y.) 194, 336 (1989); see also, J. Polchinski, Phys. Rev. Lett. 66, 397 (1991).

[7] A. Heslot, Phys. Rev. D 31, 1341 (1985); A. Ashtekar and T.A. Schilling, gr-qc/9706069, and references therein; D.C. Brody 
and L.P. Hughston, J. Geom. Phys. 38, 19 (2001); L.P. Hughston, in Twistor Theory, edited by S. Huggett (Marcel Dekker, New York, 1995); R. Cirelli, A. Mania, and L. Pizzocchero, J. Math. Phys. 31, 2891 (1990).

[8] A.M. Bloch, Phys. Lett. A 116, 353 (1986).

[9] J. Anandan and Y. Aharonov, Phys. Rev. Lett. 65, 1697 (1990); J. Anandan, Found. Phys. 21, 1265 (1991).

[10] J.P. Provost and G. Vallee, Commun. Math. Phys. 76, 289 (1980).

[11] D. Page, Phys. Rev. A 36, 3479 (1987); G. Gibbons, J. Geom. Phys. 8, 147 (1992).

[12] N.P. Landsman, Mathematical Topics Between Classical and Quantum Mechanics (Springer, Berlin, 1998), pp. 240-242 and 462-463.

[13] See, for example, A.D. Shapere and F. Wilczek, NATO Adv. Study Inst. Ser., Ser. C 5, 1 (1989).

[14] C.W. Misner, K.S. Thorne, and J.A. Wheeler, Gravitation (Freeeman, San Francisco, 1973).

[15] C.W. Misner, in Magic Without Magic, edited by J.R. Klauder (Freeman, San Francisco, 1972), pp. 441-473.

[16] T. Banks, W. Fischler, S.H. Shenker, and L. Susskind, Phys. Rev. D 55, 5112 (1997); for a review consult, W. Taylor, Rev. Mod. Phys. 73, 419 (2001).

[17] G. 't Hooft, gr-qc/9310026; L. Susskind, J. Math. Phys. 36, 6377 (1995).
[18] V. Jejjala, R.G. Leigh, and D. Minic, hep-th/0302230; see also, Phys. Lett. B 556, 71 (2003).

[19] N. Seiberg, J. High Energy Phys. 09, 003 (2000).

[20] For a more complete set of references consult, for example, M.R. Douglas, Nucl. Phys. B (Proc. Suppl.) 68, 381 (1998); J. De Boer and K. Schalm, J. High Energy Phys. 02, 041 (2003); J. de Boer, E. Gimon, K. Schalm, and J. Wijnhout, hep-th/0212250.

[21] For a recent discussion of the issue of background dependence in $\mathrm{M}$ theory consult, for instance, T. Banks, hep-th/0011255; hep-th/0306074. At present we do not see a direct relation between our approach and the arguments raised by Banks.

[22] G. 't Hooft, hep-th/0105105; hep-th/0104219.

[23] R. Penrose, in Physics Meets Philosophy at the Planck Scale, edited by C. Callender (Cambridge Univ. Press, Cambridge, 2001), pp. 290-304.

[24] V. Balasubramanian, J. de Boer, and D. Minic, gr-qc/0211003; an alternative set of arguments as well a review of other relevant references on the subject has been given by T. Jacobson, Prog. Theor. Phys. Suppl. 136, 1 (1999).

[25] A. Connes, Non-Commutative Geometry (Academic Press, New York, 1995).

[26] D. Minic and C.H. Tze (unpublished).

[27] C.W. Misner and J.A. Wheeler, Ann. Phys. (N.Y.) 2, 525 (1957). 